\title{
Novel Microscopy based approaches to study the film formation mechanism \& associated mechanical response in polymer lattices
}

\author{
Marzena Tkaczyk ${ }^{1}$, Kalin I. Dragnevski*1, Clive R. Siviour ${ }^{1}$ \& Gary Edwards ${ }^{2}$ \\ ${ }^{1}$ Laboratory for In-situ Microscopy \& Analysis, Department of Engineering Science, \\ University of Oxford, Parks Road, Oxford, OX1 3PJ, United Kingdom \\ ${ }^{2}$ Deben UK Ltd., Brickfields Business Park, Woolpit, Bury St. Edmunds, Suffolk IP30 9QS. UK
}

*corresponding author: kalin.dragnevski@eng.ox.ac.uk, phone number: 01865273098

\begin{abstract}
In this paper we present the results from novel microscopy based approaches aimed at providing further insight into the mechanism of film formation and associated mechanical response in polymer lattices. Firstly, a 'simple' methodology, combining the use of Environmental Scanning Electron Microscopy (ESEM) and a recently introduced enhanced coolstage $\left(-50\right.$ to $\left.+50^{\circ} \mathrm{C}\right)$ was successfully developed and not only used to study dynamic processes, e.g. different stages of latex film formation, but also for high resolution imaging of 'freeze-dried' structures. By using the enhanced freeze-drying capability of the system, it was also possible to preserve the structure and features of the studied system with minimum shrinkage and distortion and in the case of polymer lattices at a desired stage of film formation. Moreover, specimens can then be readily imaged, without the need of conductive coatings and at much lower chamber gas pressures, thus minimising the beam skirting effects and allowing higher resolutions to be achieved. The second and final part of our study considers the mechanical response of the studied latex dried under different conditions, with the particular emphasis on the effects of drying rate (\% Relative Humidity). Atomic Force Microscopy (AFM) force distance curve (FDC) measurements revealed that while the \% RH did not have an effect on the structures formed, it did have an effect on the adhesive properties of the studied system. It is strongly believed that the methodologies developed and used here can be applied to other material systems, including biologicals and pharmaceuticals.
\end{abstract}


Keywords: ESEM, AFM, latex, film formation, mechanical response

\section{Introduction}

Latex can be defined as a stable mixture of spherical polymer particles (whose size is larger than that of a molecule, but small enough not to be seen by the naked eye), dispersed throughout a liquid. Nowadays, synthetic polymer latexes are of major importance in a number of industrial and everyday applications and when used for its traditional applications, such as paint and/or adhesive, the latex is applied in its wet state to a surface and allowed to dry $[1,2]$. This process, also known as latex film formation, has been widely studied in both Academia and industry and is associated with the evaporation of water [3 - 6]. The film formation process can be divided into four main stages: Stage I - dilute dispersion of polymer particles in solution, Stage II - a more concentrated particle suspension with water filled interstices, Stage III ordering of deformed particles if the temperature $(T)$ is higher than the minimum film formation temperature (Tmff or MFFT) and Stage IV - formation of a homogeneous, molecularly continuous film, a direct result of inter-diffusion of polymer chains across particle boundaries if $\mathrm{T}>\mathrm{Tg}$ (glass transition temperature) [7]. These four stages are illustrated in Fig. 1. 


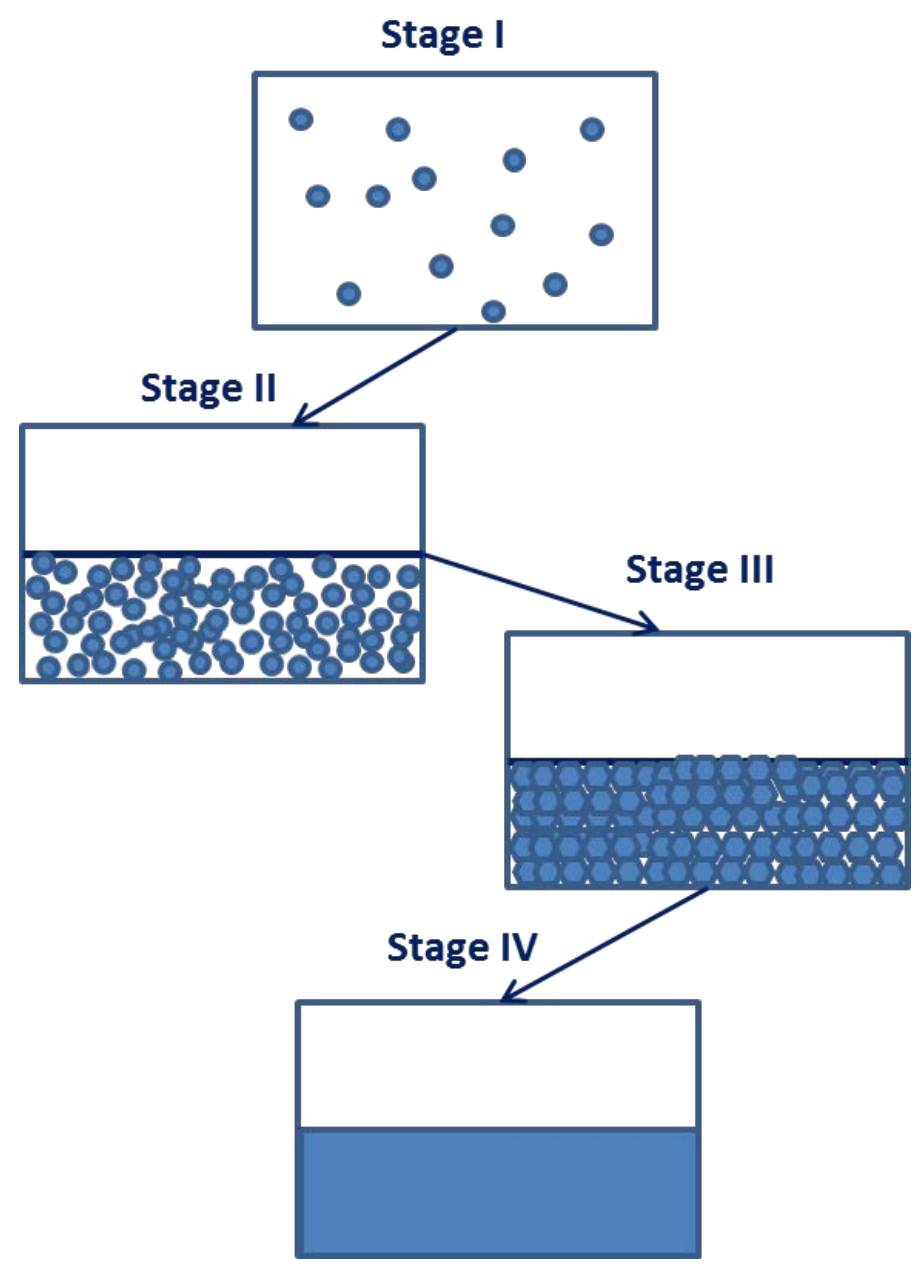

Fig. 1 Schematic representation of the four main stages of latex film formation.

However, if the drying temperature does not surpass the glass transition temperature, which is the case of the latex used in the current study, then the resulting material will dry with undeformed particles in close contact with each other.

As mentioned above, when used for its traditional applications, i.e. as paint or adhesive, this electrically insulating material is usually applied in its wet state and allowed to dry under ambient conditions. Therefore, conventional electron microscopy techniques, with their extreme drying and sample preparation requirements, are not suitable for the examination of latexes in their natural state. On the other hand, Environmental Scanning Electron Microscopy (ESEM), also referred to as "wet" or "leaky" scanning electron microscopy, offers the possibility of imaging insulating materials in their hydrated state in a gaseous environment, 
without the need of conductive coatings [8-11]. ESEM is based on the use of a multiple aperture graduated vacuum system, which allows specimens to be imaged under water vapour or other auxiliary gases, such as nitrogen or nitrous oxide [12]. Moreover, by using a correct pumpdown procedure and by controlling the temperature of the specimen, which in the ESEM is usually done by using a Peltier stage [13], dehydration can be inhibited and hence samples can be imaged hydrated. However, imaging wet samples has proved to be a challenging task and not necessarily easily repeatable and hence freeze -drying has remained the more widely used method for observing moisture containing samples. Freeze drying is a well-established process of freezing the material and sublimating the water in the material directly from the solid phase to the gas phase by reducing the surrounding pressure [14].

Alternatively, Atomic Force Microscopy (AFM) can also and has been successfully used in the study of the process of film formation $[12,13]$. The AFM technique offers further advantages to the EM approach, such as high resolution accurate surface specimen mapping at ambient conditions. AFM is generally used in three modes: contact, tapping and non-contact mode. The contact mode is based on the use of a cantilever containing a sharp tip that is in a contact with the surface of the specimen and scans it line by line. The raised and lowered features on the sample surface influence the deflection of the cantilever which is measured by a laser beam that is reflected off the cantilever and directed onto a position-sensitive photo diode. The signal from the diode is transformed into a 3D surface image. To prevent the damage of the sample and overcome problems associated with friction, adhesion or electrostatic forces the so-called tapping mode can be used. This mode is based on oscillation of the cantilever above the sample surface with a specific amplitude. During scanning, the vertically oscillating tip alternately contacts the surface and lifts off. As the oscillating cantilever contacts the surface, the cantilever oscillation is reduced due to energy loss caused by the tip contacting the surface. The reduction in oscillation amplitude is used to identify and measure surface features. 
In non-contact mode, the tip of the cantilever does not contact the samples surface at all. The resonant frequency of the cantilever is affected by atomic forces which appear between the sample and cantilever thus allowing to obtain an image of the surface. Furthermore, by monitoring the cantilever deflection (d) as a function of the vertical displacement of the piezoelectric scanner (z) it is also possible to generate a Force Distance Curve (FDC) and thus obtain valuable information of the mechanical response of the system under investigation [15]. To achieve this, laser beam is focused on the free end of the cantilever with the tip, which is approaching and retracting from the surface. The position of the reflected beam is detected by a position sensitive detector (PSD). The cantilever deflection can then be converted into force (F) using Hooke's law ( $F=-k d$, where $k$ is the cantilever spring constant).

In this research we used the above described techniques to study the film formation mechanism and investigate the effects of drying rate, i.e. humidity, on the microstructural evolution and respective mechanical response of drying polymer latex films. The results presented and discussed here form part of our long-term strategy aimed at the development and optimisation of advanced techniques for in-situ experimentation used to probe the structureproperty-composition relationship in soft systems on a macro-, micro- \& nano- scales..

\section{Materials and Methods}

The aqueous polymethylmetaclylate (PMMA) latex used in this study was supplied by the Department of Chemistry, University of Oxford and consisted of polymethylmethacrylate (PMMA) particles with diameter in the region of $<500 \mathrm{~nm}$. The latex was initially $75 \mathrm{wt} . \%$ polymer. Specimens for the film formation studies were cast directly onto standard $12.5 \mathrm{~mm}$ aluminium SEM stubs and $9.88 \mathrm{~mm}$ dish-style aluminium stubs, whereas those for the humidity studies were cast directly onto glass slides. 
The microstructural analysis was carried out for latex specimens in wet, freeze-dried and dried conditions on a Carl Zeiss Evo LS15 Variable Pressure Scanning Electron Microscope, equipped with a LaB6 gun source and a range of detectors for electron imaging. For the samples in 'wet' (partially dehydrating) and dried conditions imaging was carried out under a nitrogen atmosphere ( 10Pa), at accelerating voltages in the region of $12.5-13.5 \mathrm{kV}$ and working distances in the region of $10-15 \mathrm{~mm}$. The combination of these imaging conditions resulted in minimal beam damage of the specimens during examination. The actual specimens were mounted onto the recently developed enhanced Deben Ultracoolstage (Fig. 2), which offers an extended temperature capability $\left(-50\right.$ to $\left.+50^{\circ} \mathrm{C}\right)$, thus allowing any SEM chamber to be easily converted into what can be described as a freeze-drying facility. For the freeze-drying studies specimens were prepared following established sample preparation protocols [14].

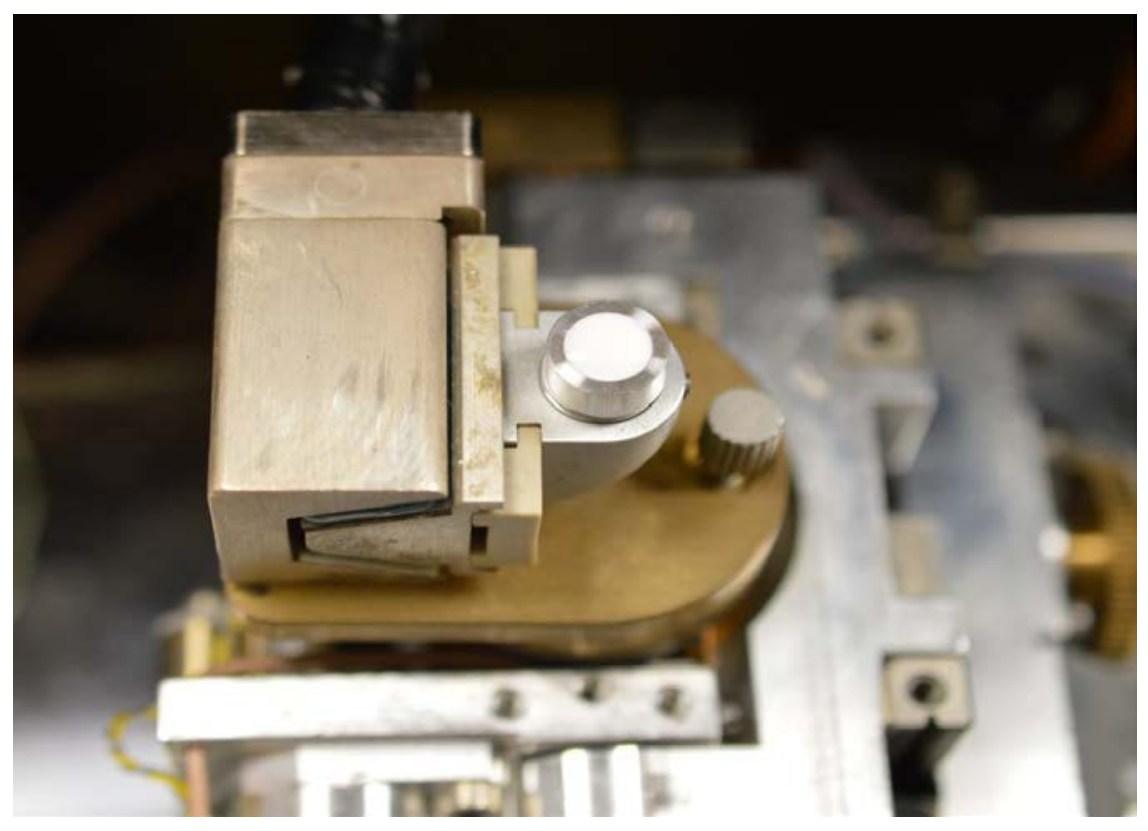

Fig. 2 Ultra Coolstage $\left(-60\right.$ to $\left.+150^{\circ} \mathrm{C}\right)$ with the latex sample

In order to study the effect of sample conditioning, i.e. \% Relative Humidity (RH), on the film formation mechanism and respective mechanical response of the latex, the samples were dried at room temperature for 48 hours in desiccators in the presence of different saturated solutions of salts that ensured conditioning at humidity levels of $0 \%\left(\mathrm{P}_{2} \mathrm{O}_{5}\right), 50 \%\left(\mathrm{Mg}\left(\mathrm{NO}_{3}\right)_{2}\right)$ 
and 90\% $\left(\mathrm{KNO}_{3}\right)$ [Error! Bookmark not defined.]. The Atomic Force Microscopy (AFM) measurements were carried out on an upgraded Vecco Dimensions 3100, equipped with a Nanoscope IIIa controller, in tapping mode, using Micromash cantilevers with a spring constant of $40 \mathrm{~N} / \mathrm{m}$. The ramp size used was $1 \mu \mathrm{m}$ with a $0.5 \mathrm{~Hz}$ for all force curves acquired.

\section{Results and Discussion}

From the images obtained under 'wet' (partially dehydrating) conditions it is clearly seen that while it was possible to observe individual particles, it is difficult to distinguish between them and any associated boundaries and/or arrangements, whether cubic or hexagonal (Fig. 3a). Better quality images were obtained from the air-dried specimens (Fig. 3b), but highresolution imaging proved to be difficult. However, subsequent freeze-drying, as expected, resulted in the observation of a well-defined and more stable (in imaging terms) structure (Fig. 4a). With the freeze-drying technique it was also possible to image individual particles and their interactions at much higher resolutions (Fig. $\mathbf{4 b}$ ), clearly demonstrating the potential advantages that this methodology offers. From the image it is also clearly seen that on this occasion the latex specimen is in Stage II of the film formation process, where partial evaporation has led to the formation of a concentrated suspension of particles, some of which are in contact with each other. By using this method it is also possible to preserve the structure and features of the studied system with minimum shrinkage and distortion and in the case of polymer lattices at a desired stage of film formation. It also reduces possibility of frost formation, which can occur during moving the sample from an external freeze-drying device to the SEM chamber [14]. Also conducting the observations of the latex specimens with ESEM eliminates the need of conductive coating and thus allows examination of the real surface of the specimen. 

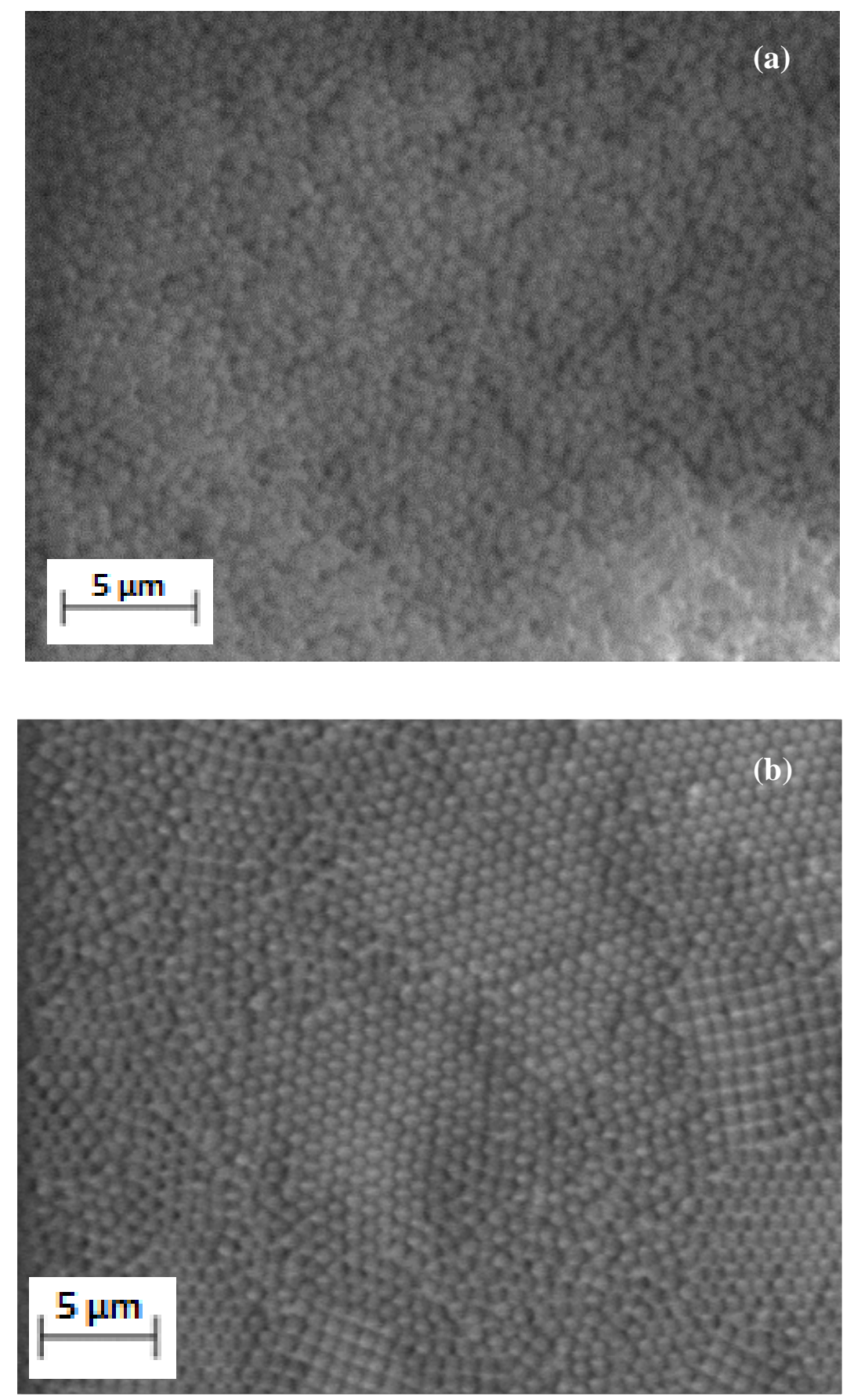

Fig. 3 ESEM images of hydrated (a) \& (b) air-dried polymethylmetaclylate latex 

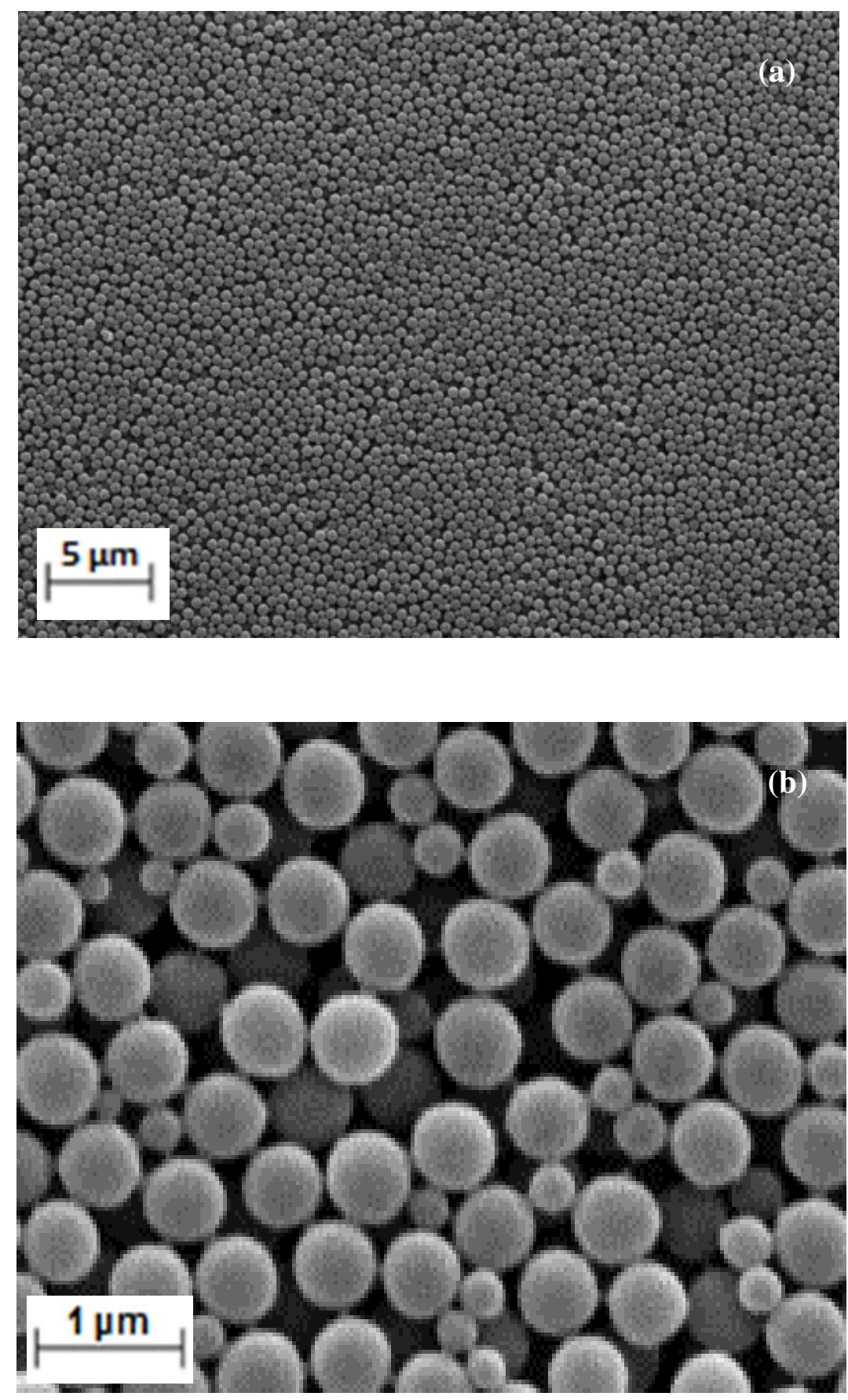

Fig. 4 ESEM image of in-situ freeze-dried polymethylmetaclylate latex at different magnifications (a - low, b - high)

Examples of typical high resolution Atomic Force Microscopy images obtained for dried latex samples are showed in Fig. 5. In all cases with different drying rates, the structure of the dried latex films consisted of randomly packed particles and although hexagonal and cubic close packed regions can be seen in the images below they are by no means a dominant 
feature of the structure of the specimen. Therefore it would appear that for this high Tg nonfilm forming PMMA based latex, humidity does not affect its drying mechanism.
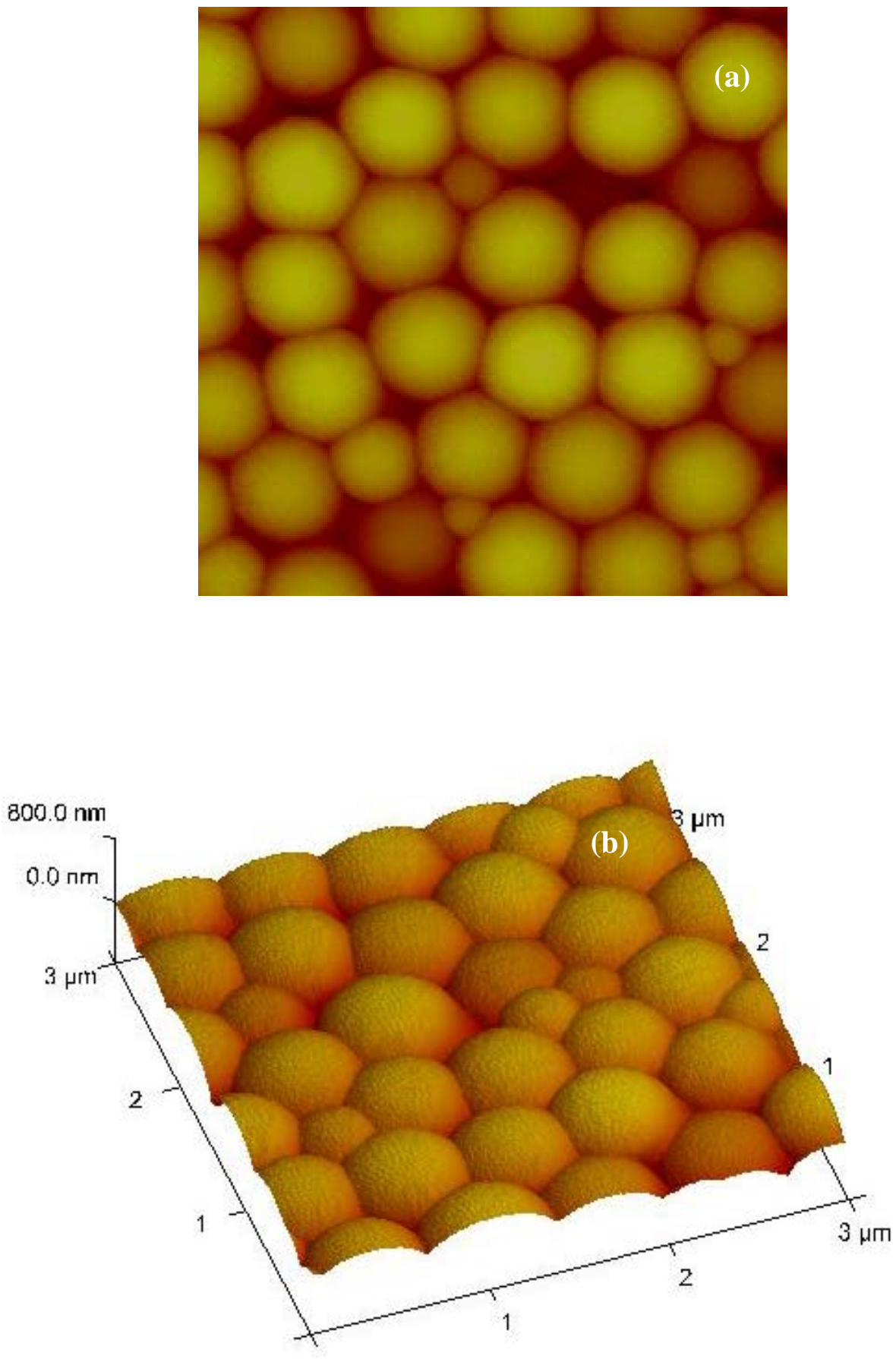

Fig. 5 Typical 2D (a) \& 3D (b) tapping mode AFM images of the dried latex specimens (2D AFM 50\% RH and 3D AFM 0\% RH) 
A typical set of Force Distance Curves obtained from the latex specimens film formed at various humidity levels is shown in Fig. 6. It can be clearly seen that the deflection varies considerably, thus indicating differences in the mechanical response of the specimens.

In the studied polymer system the deflection increases with increasing RH. As mentioned before, the force curve can be used to calculate the contact force of the tip on the sample if the spring constant, $k$, of the cantilever is known. The resultant pull-off force, which represents adhesion is plotted as a function of \% Relative Humidity and shown in Fig. 7. It is evident that the force for this particular case increases linearly and the relationship can also be represented with the equation below:

$$
F=7.18 \phi+165
$$

where $F$ is the pull off force in $\mathrm{nN}$ and $\phi$ is the relative humidity.

If drying is fast, i.e. the water contents of the latex are removed quickly, which is the case of $0 \% \mathrm{RH}$, the film formed will in essence be a structure of mechanically interlocked polymer particles and hence it will be reasonable to expect a low pull off force. At more moderate drying, an i.e. higher level of Relative Humidity, there is an increased chance to retain moisture within the films, thus presenting a much softer structure. Also, under wetter conditions it is possible that the colloidal interparticle forces involved in the process of film formation, such as Van der Walls, capillary and gravitational, play an additional role, which would result in a more adhesive film. 


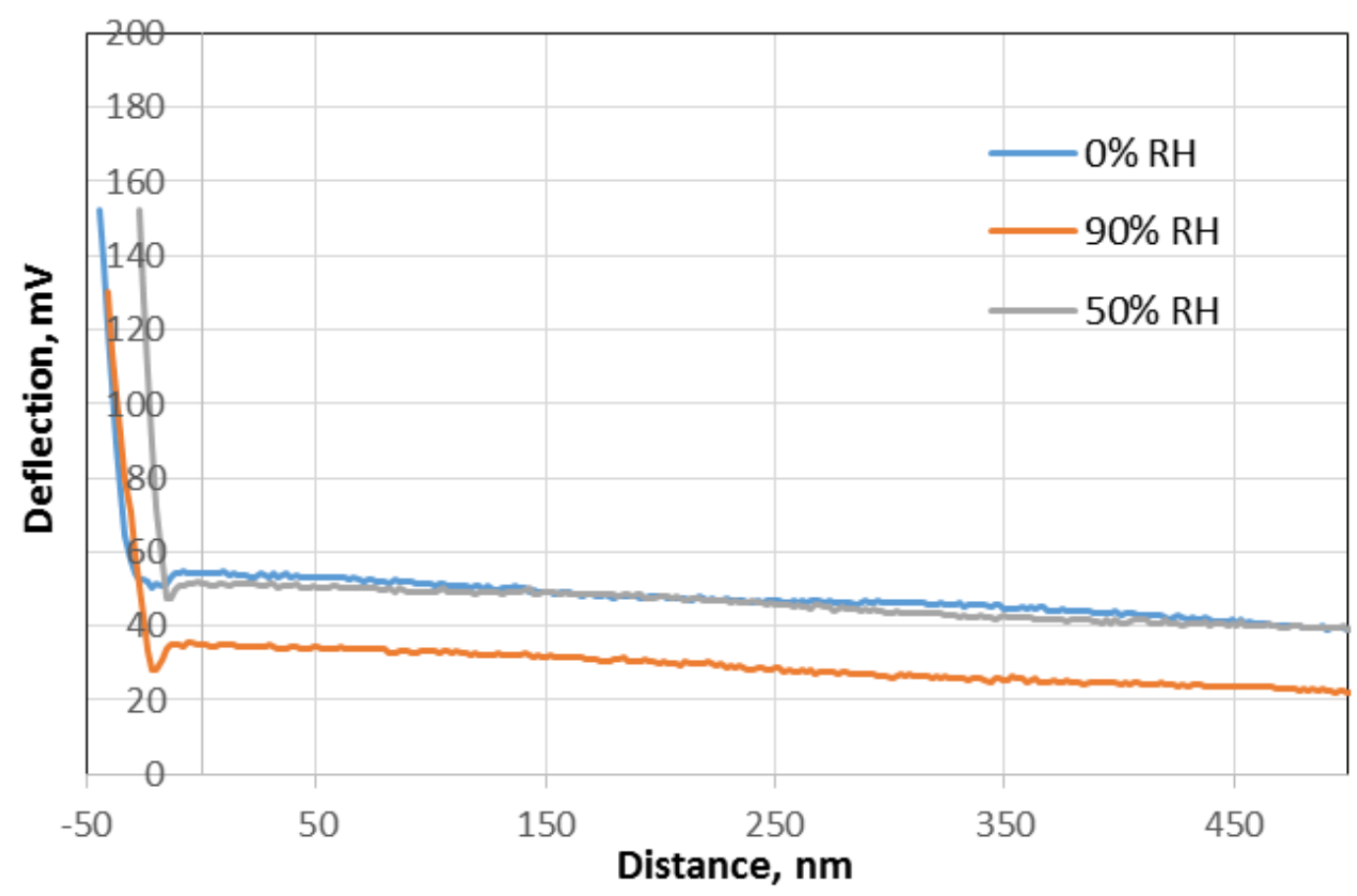

Fig. 6 Force distance curves obtained from the latex specimens film formed 0\%, 50\% and 90\% Relative Humidity

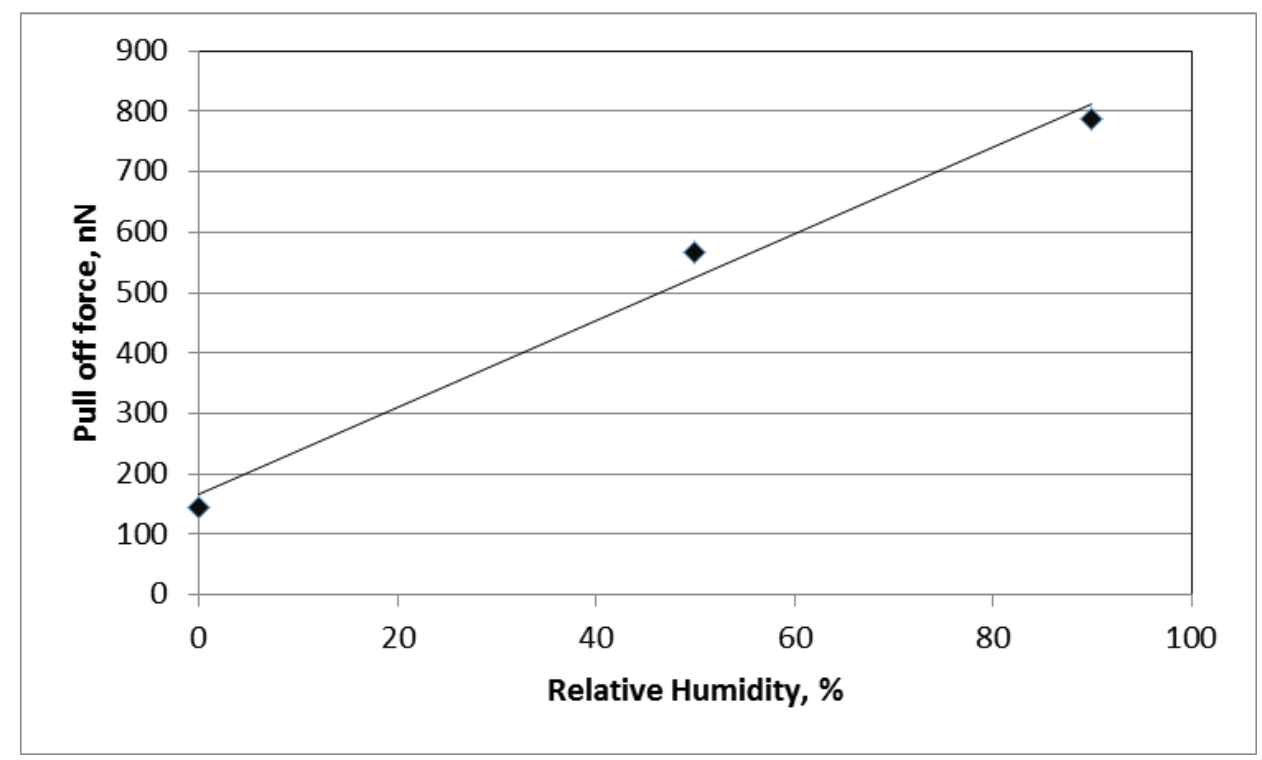

Fig. 7 Plot of pull-off force vs Relative Humidity obtained from the AFM measurements for the dried PMMA latex films 


\section{Conclusions}

In this study the PMMA latex film formation process and associated mechanical response as a function of drying rate was investigated by adopting an alternative combination of ESEM and AFM techniques. The results clearly demonstrate that when combined with an (E)SEM, the Ultra Coolstage $\left(-60\right.$ to $\left.+150^{\circ} \mathrm{C}\right)$ can not only be used to study dynamic processes, but also for high resolution imaging of freeze-dried structures; in our case PMMA latex particles. It is strongly believed that the methodology can be applied to other material systems, including biologicals and pharmaceuticals, where liquid content plays a role. Furthermore, specimens can also be readily imaged, with or without the need of conductive coatings and at much lower chamber gas pressures, thus minimising the beam skirting effects and allowing higher resolutions to be achieved.

Moreover we have also successfully used the atomic force microscopy techniques (AFM-FDC approach) to investigate the effects of humidity on the film formation mechanism and respective mechanical response. The results suggest that while the Relative Humidity did not have an effect on the structures formed, it did have an effect on the adhesive properties of the specimens. It was found that the pull-off force increases linearly with increasing humidity. The results presented and discussed here, as suggested above, form part of our long-term strategy aimed at further developing in-situ experimentation techniques used to gain further insight into the complex interaction mechanisms of polymers and colloids. 


\section{References}

[1] D.P. Sheettz, Formation of films by drying of latex, J. Appl. Polym. Sci. 9 (1965) 37593773.

[2] L. N. Butler, Ch.M. Fellows, R.G. Gilbert, Effect of surfactants used for binder synthesison the properties of latex paints, Prog. Org. Coat. 53 (2005) 112-118.

[3] Ch-L Zhao, Y. Wang, Z. Hruska, M. A. Winnik, Molecular Aspects of Latex Film Formation: An Energy-Transfer Study, Macromolecules 23 (1990) 4082-4087.

[4] P.A. Steward, J. Hearn, M.C. Wilkinson, An overview of polymer latex film formation and properties, Adv. Colloid Interface Sci. 86 (2000) 195-267.

[5] A. F. Routh, W. B. Russel, A Process Model for Latex Film Formation: Limiting Regimes for Individual Driving Forces, Langmuir 15 (1999) 7762-7773.

[6] A. F. Routh, W.B. Russe, Deformation Mechanisms during Latex Film Formation: Experimental Evidence, Ind. Eng. Chem. Res. 40 (2001), 4302-4308.

[7] O. Islam, K.I. Dragnevski, C. R. Siviour, On some aspects of latex drying - ESEM observations, Prog.Org. Coat. 75 (2012) 444-448

[8] A. Donald, The use of environmental scanning electron microscopy for imaging wet and insulating materials, Nature Materials 2.8 (2003) 511-6.

[9] M.Gretz, J.Plank, An ESEM investigation of latex film formation in cement pore solution Cement and Concrete Research 41, Issue 2 (2011) 184-190.

[10] K. Dragnevski, A. Donald, P.Taylor, M. Murray, S. Davies, E. Bone, Latex Film Formation in the Environmental Scanning Electron Microscope, Macromol. Symp. 281 (2009) $119-125$.

[11] K.I. Dragnevski, et al.: Structure-property relationship in aging acrylic latex films, Progress in organic Coatings, 65, 19-24, (2009) 
[12] M. Braga, C.A. Rodrigues Costa, C.A.P. Leite, F. Galembeck, Scanning Electric Potential Microscopy Imaging of Polymer Latex Films: Detection of Supramolecular Domains with Nonuniform Electrical Characteristics, J. Phys. Chem. B 105 (2001) 3005-3011.

[13] M. Meincken, R. D. Sanderson, Determination of the influence of the polymer structure and particle size on the film formation process of polymers by atomic force microscopy, Polymer 43 (2002) 4947-4955.

[14] https://www.quorumtech.com/quorum-product/k750x-peltier-cooled-em-freeze-dryer [15] Z.Y., Dragnevska, et al.; Application of novel thermal AFM Scanning probe techniques in the adhesion studies of E.coli cells, Micro and Nanosystems, 4, 86-91, (2012). 